Article

\title{
Erbium-Doped Amorphous Carbon-Based Thin Films: A Photonic Material Prepared by Low-Temperature RF-PEMOCVD
}

\author{
Hui-Lin Hsu ${ }^{1}$, Keith R. Leong ${ }^{1}$, I-Ju Teng ${ }^{2,3}$, Michael Halamicek ${ }^{1}$, Jenh-Yih Juang ${ }^{2,3}$, \\ Sheng-Rui Jian ${ }^{4}$, Li Qian ${ }^{1, *}$ and Nazir P. Kherani ${ }^{1,5}, *$
}

1 Department of Electrical and Computer Engineering, University of Toronto,

Toronto, ON M5S 3G4, Canada; E-Mails: huilin.hsu@mail.utoronto.ca (H.-L.H.);

keith.leong@mail.utoronto.ca (K.R.L.); michael.halamicek@mail.utoronto.ca (M.H.)

2 Centre for Interdisciplinary Science, National Chiao Tung University, Hsinchu 30010, Taiwan;

E-Mails: ijteng@nctu.edu.tw (I.J.T.); jyjuang@nctu.edu.tw (J.-Y.J.)

3 Department of Electrophysics, National Chiao Tung University, Hsinchu 30010, Taiwan

4 Department of Materials Science and Engineering, I-Shou University, Kaohsiung 84001, Taiwan;

E-Mail: srjian@gmail.com

5 Department of Materials Science and Engineering, University of Toronto,

Toronto, ON M5S 3E4, Canada

* Authors to whom correspondence should be addressed; E-Mails: 1.qian@utoronto.ca (L.Q.); kherani@ecf.utoronto.ca (N.P.K.); Tel.: +1-416-946-5517 (L.Q.); +1-416-946-7372 (N.P.K.).

Received: 26 November 2013; in revised form: 17 February 2014 / Accepted: 19 February 2014 / Published: 27 February 2014

\begin{abstract}
The integration of photonic materials into CMOS processing involves the use of new materials. A simple one-step metal-organic radio frequency plasma enhanced chemical vapor deposition system (RF-PEMOCVD) was deployed to grow erbium-doped amorphous carbon thin films (a-C:(Er)) on $\mathrm{Si}$ substrates at low temperatures $\left(<200{ }^{\circ} \mathrm{C}\right)$. A partially fluorinated metal-organic compound, tris(6,6,7,7,8,8,8-heptafluoro-2,2-dimethyl-3,5octanedionate) Erbium(+III) or abbreviated $\operatorname{Er}(\text { fod })_{3}$, was incorporated in situ into a-C based host. Six-fold enhancement of Er room-temperature photoluminescence at $1.54 \mu \mathrm{m}$ was demonstrated by deuteration of the a-C host. Furthermore, the effect of RF power and substrate temperature on the photoluminescence of a-C:D(Er) films was investigated and analyzed in terms of the film structure. Photoluminescence signal increases with increasing $\mathrm{RF}$ power, which is the result of an increase in $[\mathrm{O}] /[\mathrm{Er}]$ ratio and the respective erbium-oxygen coordination number. Moreover, photoluminescence intensity decreases
\end{abstract}


with increasing substrate temperature, which is attributed to an increased desorption rate or a lower sticking coefficient of the fluorinated fragments during film growth and hence [Er] decreases. In addition, it is observed that Er concentration quenching begins at $\sim 2.2$ at $\%$ and continues to increase until 5.5 at\% in the studied a-C:D(Er) matrix. This technique provides the capability of doping Er in a vertically uniform profile.

Keywords: RF-PEMOCVD; erbium metal-organic compound; deuterated amorphous carbon (a-C:D); fluorination

\section{Introduction}

Incorporating optical technologies into microelectronic devices has been researched as a viable solution to overcome the speed bottlenecks associated with the ever shrinking device features [1]. The optical interconnect is able to transfer and process data at rates that are orders of magnitude higher than traditional electronic technologies, both within a Si chip and in chip-to-chip communications. In order to completely avail optical technologies, it is imperative to develop silicon compatible materials that enable light generation, guiding, switching, detection, modulation and amplification. To realize the co-existence of electrical and optical functions on the same Si chip platform, it is essential to develop Si compatible photonic materials. Further, the materials processing temperatures being below $400{ }^{\circ} \mathrm{C}$ is highly desirable [2] as this would meet the Si back end-of-line (BEOL) requirements in the current $\mathrm{Si}$ integrated circuit (IC) fabrication technology.

Crystalline silicon is not readily amenable for light emission due to its inherent indirect bandgap characteristic. Although, major breakthroughs in this field include the observation of an optical gain in Si nanocrystals [3], development of a Si Raman laser [4], electroluminescence for a Si diode [5], Si nanocrystal field-effect-transistors [6], and realization of a high-speed Si electro-optic modulator [7], these approaches have efficiency limitations. As well, the materials or the processing methods proposed require considerable development to ensure compatibility with current Si technology.

Ion implantation of light-emitting impurities, such as erbium (Er), into a variety of silica-based [8,9], ceramic [9], and Si-based [9,10] thin film hosts, has been a leading technique availed by the scientific community to efficiently produce photons from $\mathrm{Si}$. The advantage of this approach is that standard $\mathrm{Si}$ technology can be deployed to introduce $\mathrm{Er}$ as a dopant. In addition, $\mathrm{Er}^{3+}$ ions can emit photons at $1.5 \mu \mathrm{m}$, which is a strategic wavelength for telecommunications due to a minimum in the absorption of silica fibers. However, high processing temperatures are required to grow silica-based $\left(\mathrm{SiO}_{2}\right.$, phosphosilicate, borosilicate) and ceramic $\left(\mathrm{Al}_{2} \mathrm{O}_{3}, \mathrm{Y}_{2} \mathrm{O}_{3}, \mathrm{LiNbO}_{3}\right)$ thin film hosts. These are incompatible with Si BEOL fabrication processes. Also, high temperature $\left(>700{ }^{\circ} \mathrm{C}\right)$ post-deposition annealing processing is necessary to eliminate the ion implantation-induced damage, optically activate the $\mathrm{Er}^{3+}$ ions, and enhance the photoluminescence lifetime or quantum efficiency [9]. However, in many cases, high temperature annealing is not sufficient to remove the defects. For instance, ion implantation-induced defects still exist in high Er fluence implanted borosilicate glass. Despite subjecting the samples to a post-deposition annealing step, additional quenching sites coupled to $\mathrm{O}-\mathrm{H}$ bonds are created. For crystalline Si based hosts, photoluminescence is severely quenched at room temperature as a result 
of the strong non-radiative processes that compete with the radiative Er de-excitation. Co-implantation of additional $\mathrm{O}$ atoms (highly preferred) reduces the Er segregation/precipitation by forming $\mathrm{Er}-\mathrm{O}$ complexes, and increases the fraction of active $\mathrm{Er}^{3+}$ ions. To overcome the quenching issue, a $\mathrm{SiO}_{2}$ matrix containing Er-doped Si nanocrystals has been applied. The Si nanocrystals act as efficient sensitizers, which is attributed to an effective Er excitation cross section that is more than two orders of magnitude larger compared to the Er resonant absorption of a photon [11]. However, high temperature post-deposition annealing processing is inevitable in order to form $\mathrm{Si}$ nanocrystals inside a $\mathrm{SiO}_{2}$ matrix. High Er concentration and lower temperature quenching can be achieved in hydrogenated amorphous $\mathrm{Si}$ (a-Si:H) and/or porous $\mathrm{Si}$. This is achieved by using low temperature processing methods, such as low pressure chemical vapor deposition (LPCVD) or plasma enhanced chemical vapor deposition (PECVD), both of which are Si BEOL compatible. Nevertheless, high temperature post-deposition annealing is required in order to observe photoluminescence in Er doped a-S:H and/or porous Si films.

Although extensive research on Er-implanted silicon and silica-based materials has been performed, Er doping in an amorphous carbon based host has received little attention [12-16]. Hydrogenated amorphous carbon (a-C:H) films, for example grown by a low-temperature PECVD method, exhibits compatible processing parameters with current CMOS fabrication technology. This facilitates integration and allows for reproducible, low-cost films. In addition, a-C based films possess a number of outstanding properties such as high chemical resistance, mechanical hardness, biocompatibility [17], and transparency in the infrared [18,19]. Due to their excellent tribiological properties, a-C:H films are widely used as protective coatings for hard disks and magnetic media, machine parts, optical windows and fibers, and other surfaces [20]. The specific properties of a-C:H films can be tailored over a wide range by adjusting the amount of $\mathrm{sp}^{3}$ and $\mathrm{sp}^{2}$ hybridized carbon, and the incorporated hydrogen content in the film via various deposition parameters and methods [19]. Moreover, these films can be deposited uniformly over a large area. These unique and versatile properties provide an impetus to utilize PECVD a-C films for specific optoelectronic applications.

The first reported demonstration of room-temperature photoluminescence (PL) from Er at $1.54 \mu \mathrm{m}$ in a-C:H(Er) thin films was published in 2002 [12]. a-C:H(Er) films were deposited by magnetron sputtering of a graphite target that was partially covered by Er platelets in an $\mathrm{Ar} / \mathrm{C}_{6} \mathrm{H}_{12}$ atmosphere. The Er concentration in the a-C:H(Er) films could be changed from 0.15 at $\%$ to 1.2 at $\%$. However, the PL intensity was relatively low. This was caused by the non-radiative relaxation pathway induced by $\mathrm{C}-\mathrm{H}$ vibrations and the low optical band gap $(\sim 0.5 \mathrm{eV})$ of the sample [15]. In this deposition technique, the Er concentration depends highly on the degree of magnetron sputtering of the Er/graphite target. Accordingly, high Ar ion energy and flux are required to achieve high Er concentration. However, this causes a high concentration of $s p^{2}$ carbon and a low optical bandgap. The incorporation of an $\mathrm{Er}$ metal-organic compound into a carbon layer by the radio frequency PECVD method was demonstrated by Prajzlera et al. [14] in 2003, however, no PL spectra were presented. In 2009, Tsai et al. [16] grew a-C:H(Er) and a-C:D(Er) films via in situ thermal evaporation of the tris(2,2,6,6-tetramethyl-3-5 heptanedionato) erbium(+III), or Er(tmhd) $)_{3}$, compound in a DC saddle-field PECVD system. A PL signal was observed from both a-C:H(Er) and a-C:D(Er) films. However, the relative PL intensity from a-C:H(Er) was at least 10 times lower than from a-C:D(Er), attributed to the optical quenching from the highly abundant $\mathrm{C}-\mathrm{H}$ bonds. Further, the $\mathrm{Er}(\mathrm{tmhd})_{3}$ metal-organic compound contains a high 
percentage of $\mathrm{C}-\mathrm{H}$ bonds, 58.76 at $\%$. Hence, this precursor is inherently inefficient at promoting $\mathrm{Er}^{3+}$ photoluminescence.

In this work, the feasibility of in situ growth of Er-doped a-C based thin films $(\mathrm{a}-\mathrm{C}(\mathrm{Er}))$ at low temperatures $\left(<200{ }^{\circ} \mathrm{C}\right)$ by simple occlusion of a metal-organic in a radio frequency plasma enhanced chemical vapor deposition (RF-PEMOCVD) system is investigated. The properties of the a-C host film and the incorporated Er concentration are independently controlled. The effect of RF power and substrate temperature on the photoluminescence of a-C(Er) films is investigated. The film structure, incorporated Er concentration, and the effect of the change in optical properties of host a-C are also discussed.

\section{Results and Discussion}

\subsection{Photoluminescence Enhancement by Deuteration of a-C host}

In the Er metal-organic compound, non-radiative deactivation channels exist near the active $\mathrm{Er}^{3+}$ ions due to the surrounding ligands. Any additional $\mathrm{C}-\mathrm{H}$ and $\mathrm{O}-\mathrm{H}$ bonds from the host material would cause additional quenching of the $\mathrm{Er}^{3+}$ luminescence. Thus, it is not surprising that there are only a few publications [12-16] which demonstrate the photoluminescence of Er complexes in hydrogenated amorphous carbon $(\mathrm{a}-\mathrm{C}: \mathrm{H})$, or other carbon-based related materials such as polymers [21,22]. To suppress the quenching effect from the $\operatorname{Er}(\text { fod })_{3}$ ligands and the host material, the precursor gas used to deposit the host a-C was changed from methane $\left(\mathrm{CH}_{4}\right)$ to deuterated methane $\left(\mathrm{CD}_{4}\right)$. The resultant PL spectrum is depicted in Figure 1a. The room temperature PL spectra centered at $1540 \mathrm{~nm}$ corresponds to the ${ }^{4} I_{13 / 2}$ to ${ }^{4} I_{15 / 2}$ electronic transition of $\mathrm{Er}^{3+}$ ions. The spectral width of the emission band is due to inhomogeneous and homogeneous broadening in addition to Stark splitting of the $\mathrm{Er}^{3+}$ excited and ground states. The PL peak is wider than those of other Er-implanted silicate glasses [9], indicating that $\mathrm{Er}^{3+}$ possesses a variety of local bonding environments in the a-C matrix. Its $65 \mathrm{~nm}$ full width at half-maximum (FWHM) suggests the potential of enabling a wide gain band width for optical amplification. It is observed that the intensity of PL is enhanced by a factor of six with the same Er concentration in the a-C:D matrix. This confirms that Er luminescence is enhanced through the substitution of $\mathrm{H}$ with $\mathrm{D}$ in the a-C host samples prepared by the RF-PEMOCVD method.

The enhancement of $\mathrm{PL}$ is a result of the weaker interaction strength between $\mathrm{Er}^{3+}$ and the $\mathrm{C}-\mathrm{D}$ and $\mathrm{O}-\mathrm{D}$ third harmonic vibrations, compared to the interaction strength between $\mathrm{Er}^{3+}$ and the $\mathrm{C}-\mathrm{H}$ and $\mathrm{O}-\mathrm{H}$ second harmonic vibrations. The $\mathrm{C}-\mathrm{H}$ and $\mathrm{O}-\mathrm{H}$ second harmonic vibrations $\left(5900\right.$ and $6900 \mathrm{~cm}^{-1}$, respectively) approximately match $(\sim 1500 \mathrm{~nm})$ the radiative transition from the first excited state ${ }^{4} I_{13 / 2}$ to the ground state ${ }^{4} I_{15 / 2}$ in $\mathrm{Er}^{3+}$ ions $\left(\sim 6500 \mathrm{~cm}^{-1}\right)$ [23] as seen in Figure 2. Accordingly, if excited $\mathrm{Er}^{3+}$ ions are disturbed nearby $\mathrm{C}-\mathrm{H}$ and $\mathrm{O}-\mathrm{H}$ oscillators, a non-radiative transition transpires. By applying the undistorted oscillator model [24], the transition probability between $\mathrm{Er}^{3+}$ and the vibrational modes of the host a-C:D significantly decreases. Furthermore, by directly comparing the FTIR spectra of the host $\mathrm{a}-\mathrm{C}: \mathrm{H}$ and a-C:D films, shown in Figure $1 \mathrm{~b}$, the impact of the host a-C materials on $\mathrm{Er}^{3+} \mathrm{PL}$ quenching can be clearly seen. Deuteration of the host a-C effectively shifts the absorption of the first vibrational mode $(v=1)$ to lower wavenumber (longer wavelength) as seen in Figure 2. Also, the magnitude of the absorption has decreased by at least $50 \%$ as indicated in Figure 1b. Moreover, the higher harmonic 
vibrational modes are much weaker, implying much lower absorption of $\mathrm{C}-\mathrm{D} x$ in the third harmonic $(v=3)$ than that of $\mathrm{C}-\mathrm{H} x$ in the second harmonic $(v=2)$. Thus, this leads to an increase in the efficiency of the PL at $1540 \mathrm{~nm}$ for a-C:D (Er) films.

Figure 1. (a) Comparison of PL intensity of a-C:D(Er: 3.92 at\% ) and a-C:H(Er: 3.92 at \%) films prepared using RF power of $40 \mathrm{~W}$, precursor gas flow rate of $40 \mathrm{sccm}$, deposition pressure of 120 mTorr, substrate temperature of $80{ }^{\circ} \mathrm{C}$, and $\operatorname{Er}(\mathrm{fod})_{3}$ powder evaporation temperature of $150{ }^{\circ} \mathrm{C}$; (b) Comparison of FTIR spectra of the a-C:D and a-C:H host films free of Er metal-organic prepared under otherwise identical deposition conditions.

(a)
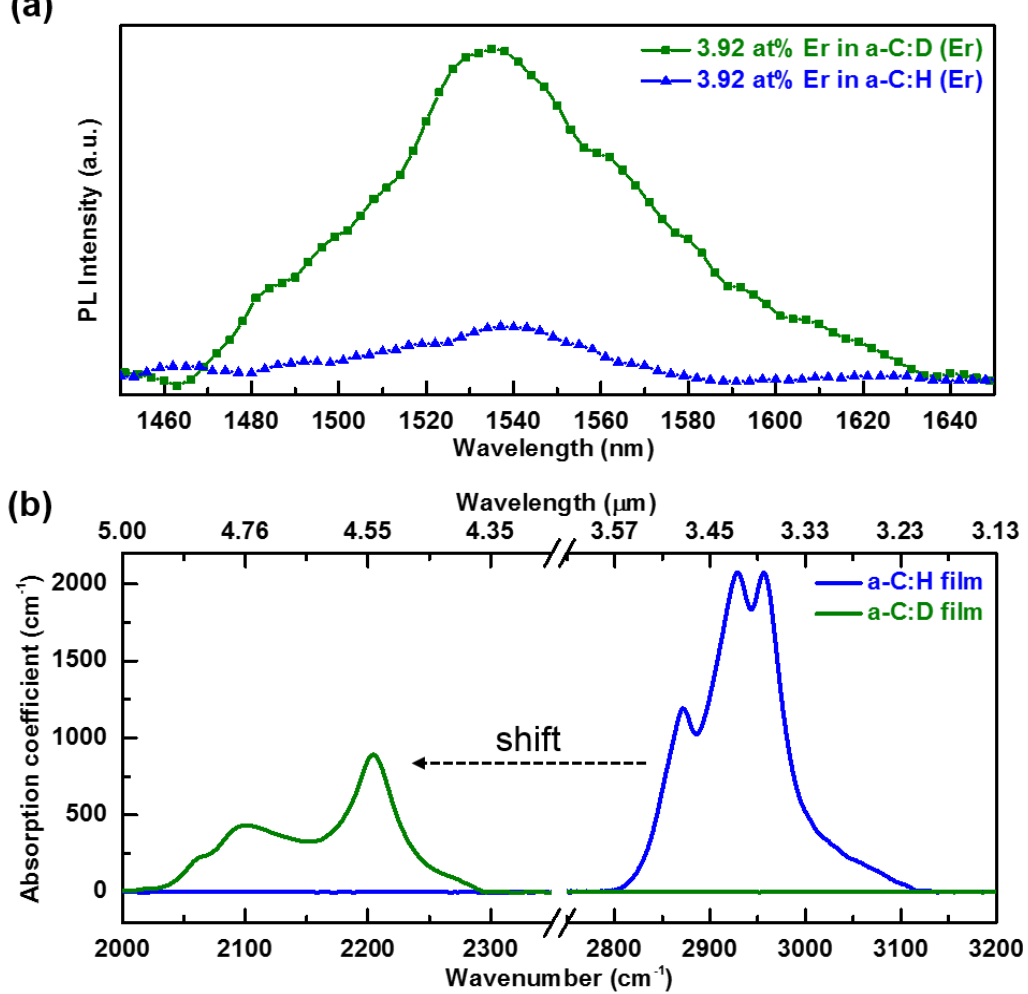

Figure 2. Illustration of energy levels of vibrational modes in hydrogen and deuterium containing organic media. Reprinted/Reproduced with permission from [23]. American Institute of Physics 1974 AIP Publishing.

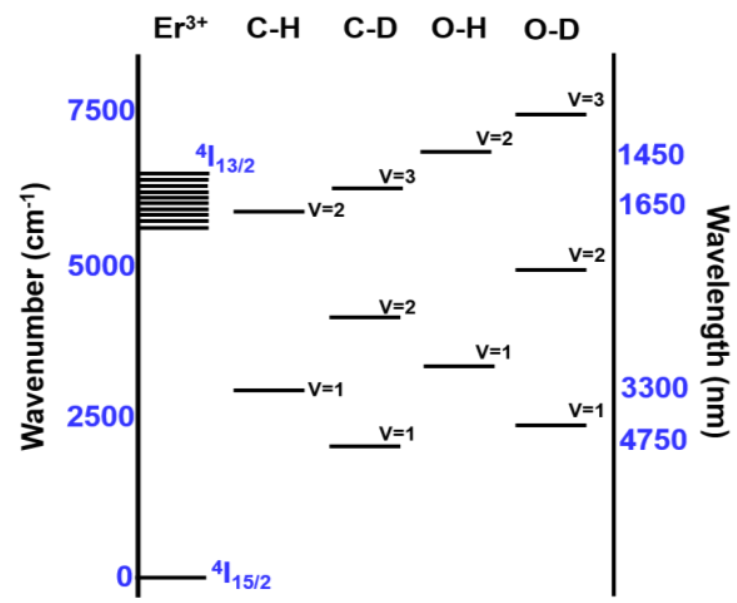




\subsection{Effect of RF Power and Substrate Temperature on a-C:D(Er) Film}

Two key parameters that are found to influence the concentration of Er occluded in the a-C:D(Er) film are the RF power and substrate temperature. Table 1 lists the ratios of the atomic concentrations and the relative (and absolute) atomic concentrations of the relevant elements in the as-received stoichiometric $\operatorname{Er}(\text { fod })_{3}$ compound, thermally evaporated (abbreviated $\left.\mathrm{TE}\right) \operatorname{Er}(\mathrm{fod})_{3}$, a-C:H(Er), and in the seven a-C:D(Er) films deposited under varying conditions as determined from XPS measurements. In comparing the a-C:D(Er) film deposited at an $\mathrm{RF}$ power of $40 \mathrm{~W}$ to the stoichiometric $\operatorname{Er}(\text { fod })_{3}$ compound, a few results are observed. The $[\mathrm{O}] /[\mathrm{C}]$ ratios are very similar, $\sim 0.19$ in the a-C:D(Er) film compared to $\sim 0.20$ in the stoichiometric $\mathrm{Er}(\mathrm{fod})_{3}$. Meanwhile, the $[\mathrm{O}] /[\mathrm{Er}]$ and $[\mathrm{C}] /[\mathrm{Er}]$ ratios are approximately $4.5 \times$ smaller in the film, while the $[\mathrm{F}] /[\mathrm{Er}]$ ratio is $2.3 \times$ smaller in the film. This suggests that Er incorporation is being promoted in the film. Moreover, since the $[\mathrm{F}] /[\mathrm{C}]$ ratio is $2 \times$ larger in the film, fluorine incorporation is also being promoted. Clearly, the plasma environment causes significant dissociation of the $\mathrm{Er}$ (fod) $)_{3}$ compound. The loss of $\mathrm{C}$ and $\mathrm{O}$ atoms is attributed to the loss of large $\mathrm{C}_{x} \mathrm{O}_{y}$ (or deuterated $\mathrm{C}_{x} \mathrm{O}_{y}$ ) fragments. The increase in the $\mathrm{F}$ concentration is due to the selective incorporation of $\mathrm{C}_{m} \mathrm{~F}_{n}$ fragments.

The Er concentration in a-C:D(Er) decreases with increasing RF power as shown in Table 1. This is evident from the increasing $[\mathrm{O}] /[\mathrm{Er}],[\mathrm{F}] /[\mathrm{Er}]$, and $[\mathrm{C}] /[\mathrm{Er}]$ ratios. The $[\mathrm{C}]$ increases with increasing $\mathrm{RF}$ power. This is observed through the increase in the $[\mathrm{C}] /[\mathrm{Er}]$ ratio and decrease in the $[\mathrm{O}] /[\mathrm{C}]$ and $[\mathrm{F}] /[\mathrm{C}]$ ratios. The $[\mathrm{O}]$ increases when compared to the $[\mathrm{F}]$ with increasing $\mathrm{RF}$ power, as seen through the increase in the $[\mathrm{O}] /[\mathrm{F}]$ ratio. The decrease in the relative $\mathrm{Er}, \mathrm{F}$, and $\mathrm{O}$ concentrations relative to $[\mathrm{C}]$ with increasing $\mathrm{RF}$ power is due to the increase in the plasma density. As the RF power increases, the dissociation of the precursors $\left(\mathrm{CD}_{4}\right.$ and $\left.\operatorname{Er}(\mathrm{fod})_{3}\right)$ increases. Thus, more $\mathrm{C}$ is being incorporated via $\mathrm{CD}_{4}$. The relative $[\mathrm{F}]$ is still quite large, 29 at $\%$ for the $60 \mathrm{~W}$ sample, which is considered to aid the PL efficiency by further reducing the $\mathrm{C}-\mathrm{H}$ quenching as discussed below in the Section 3.2. Also, note the decrease in the relative [Er], from 5.5 at $\%$ at $40 \mathrm{~W}$ to 2.8 at $\%$ at $60 \mathrm{~W}$. Despite this decrease, the $[\mathrm{O}] /[\mathrm{Er}]$ ratio increases from 1.31 to 2.38 as observed in Table 1 . The increase in $[\mathrm{O}] /[\mathrm{Er}]$ ratio is considered to increase the number of erbium-oxygen bonded complexes, which are responsible for the increase in the PL signal with increasing RF power as seen in Figure $3 a$. 
Table 1. Ratios of atomic concentrations and relative/absolute atomic concentrations of relevant elements in as-received stoichiometric Er(fod) 3 compound, thermally evaporated (TE) $\operatorname{Er}(\text { fod })_{3}$, and in seven a-C:D(Er) films deposited under varying conditions as determined from XPS measurements.

\begin{tabular}{|c|c|c|c|c|c|c|c|c|c|c|c|c|}
\hline Sample & $\begin{array}{c}\text { RF } \\
\text { Power } \\
\text { (W) }\end{array}$ & $\begin{array}{c}\text { Substrate } \\
\text { Temperature } \\
\left({ }^{\circ} \mathbf{C}\right)\end{array}$ & C at\% & Er at\% & F at $\%$ & $\mathrm{O}$ at $\%$ & {$[\mathrm{O}] /[\mathrm{Er}]$} & {$[\mathbf{F}] /[\mathbf{E r}]$} & {$[\mathrm{C}] /[\mathbf{E r}]$} & {$[\mathrm{O}] /[\mathrm{C}]$} & {$[\mathbf{F}] /[\mathbf{C}]$} & {$[\mathrm{O}] /[\mathrm{F}]$} \\
\hline $\operatorname{Er}(\text { fod })_{3}$ & - & - & 34.1 & 1.1 & 23.9 & 6.8 & 6.00 & 21.00 & 30.00 & 0.20 & 0.70 & 0.29 \\
\hline $\mathrm{TE} \operatorname{Er}(\text { fod })_{3}$ & - & - & 71.3 & 1.0 & 21.5 & 6.3 & 6.33 & 21.74 & 72.08 & 0.09 & 0.30 & 0.29 \\
\hline $\mathrm{a}-\mathrm{C}: \mathrm{H}(\mathrm{Er})$ & 40 & 80 & 54.6 & 3.9 & 35.4 & 6.0 & 1.54 & 9.08 & 14.00 & 0.11 & 0.65 & 0.17 \\
\hline 1 & 40 & 80 & 36.9 & 5.5 & 50.5 & 7.1 & 1.31 & 9.26 & 6.75 & 0.19 & 1.37 & 0.14 \\
\hline 2 & 50 & 80 & 52.3 & 4.2 & 37.4 & 6.1 & 1.47 & 9.02 & 12.60 & 0.12 & 0.72 & 0.16 \\
\hline 3 & 60 & 80 & 61.5 & 2.8 & 29.0 & 6.7 & 2.38 & 10.33 & 21.88 & 0.11 & 0.47 & 0.23 \\
\hline 4 & 60 & 90 & 77.9 & 1.4 & 5.4 & 12.3 & 8.81 & 3.85 & 55.66 & 0.16 & 0.07 & 2.29 \\
\hline 5 & 60 & 100 & 79.6 & 1.1 & 4.0 & 15.3 & 13.83 & 3.60 & 72.14 & 0.19 & 0.05 & 3.84 \\
\hline 6 & 60 & 120 & 85.7 & 0.7 & 2.4 & 11.2 & 15.86 & 3.46 & 121.58 & 0.13 & 0.03 & 4.58 \\
\hline 7 & 60 & 150 & 95.9 & 0.2 & 0.4 & 3.5 & 22.78 & 2.37 & 618.45 & 0.04 & 0.00 & 9.61 \\
\hline
\end{tabular}


Figure 3. (a) The $[\mathrm{O}] /[\mathrm{Er}]$ ratio (black triangle) and normalized PL peak intensity (blue triangle) as a function of the applied RF power with a substrate temperature of $80{ }^{\circ} \mathrm{C}$; (b) The Er concentration (solid black circle) and normalized PL peak intensity (solid blue circle) as a function of the substrate temperature with an RF power of $60 \mathrm{~W}$. The normalized $\mathrm{PL}$ peak intensity is shown to depend critically on the (c) $[\mathrm{O}] /[\mathrm{Er}] \mathrm{ratio}$; and (d) $[\mathrm{Er}]$. The lines are guides to the eye.

(a)

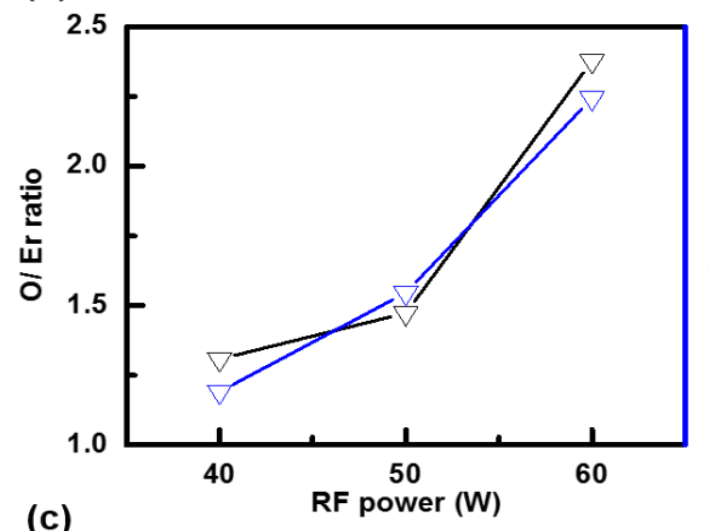

(c)

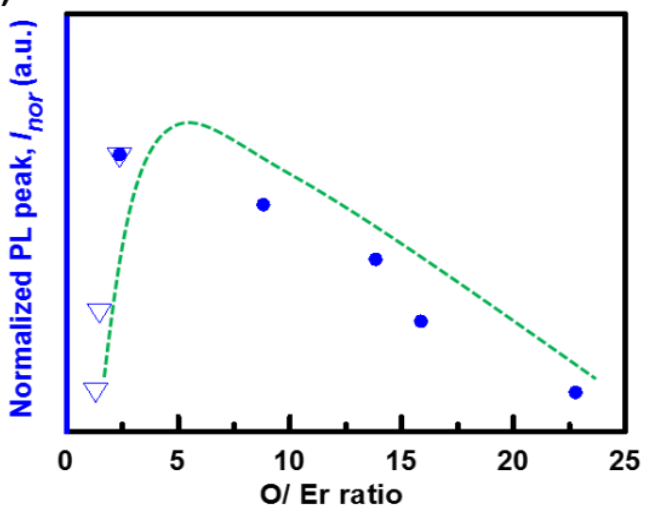

(b)

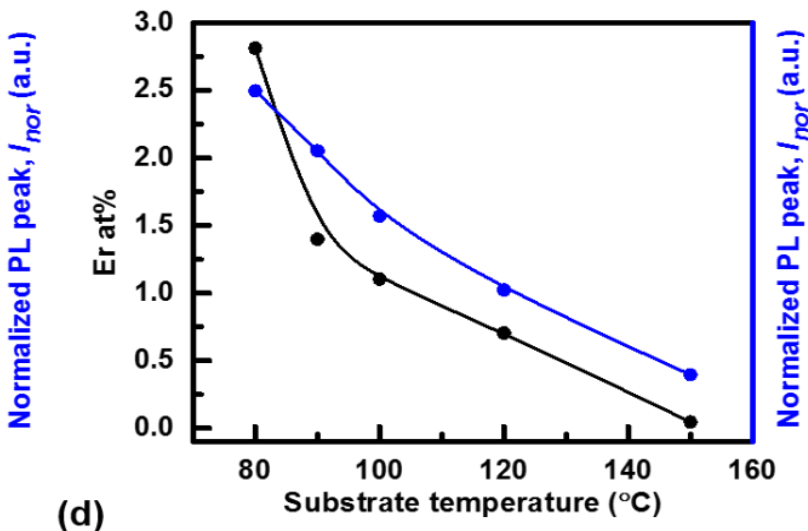

(d)

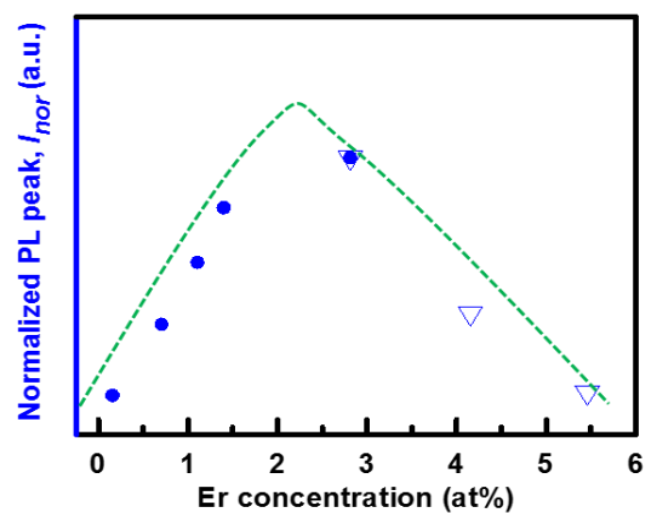

Another means of controlling the a-C:D(Er) film structure, and hence the PL efficiency, is through the temperature of the growth surface. The $\operatorname{Er}(\text { fod })_{3}$ evaporation temperature is $150{ }^{\circ} \mathrm{C}$. Hence, the temperature of the substrate will affect the relative sticking coefficient of the Er metal-organic and its associated molecular fragments. Analyzing the XPS spectra for the films grown as the substrate temperature was varied from 80 to $150{ }^{\circ} \mathrm{C}$ yields a number of results. The [F] decreases rapidly with increasing substrate temperature. This is seen through the increasing $[\mathrm{O}] /[\mathrm{F}]$ ratio and the decreasing $[\mathrm{F}] /[\mathrm{Er}]$ and $[\mathrm{F}] /[\mathrm{C}]$ ratios. The $[\mathrm{Er}]$ is also decreasing with increasing substrate temperature as shown in Figure 3b. This is evident from the increase in the $[\mathrm{O}] /[\mathrm{Er}]$ and $[\mathrm{C}] /[\mathrm{Er}]$ ratios. In examining the $[\mathrm{O}] /[\mathrm{C}]$ ratio, the $[\mathrm{O}]$ increases, peaks at a substrate temperature of about $100{ }^{\circ} \mathrm{C}$, then decreases as the substrate temperature increases. The considerable loss of $F$ is associated with a drop in the deposition of fluorinated $\operatorname{Er}(\mathrm{fod})_{3}$ molecular fragments. This is presumably due to either an increased desorption rate or a lower sticking coefficient of the fluorinated fragments. This is supported by the rise in the $[\mathrm{O}] /[\mathrm{C}]$ ratio, which implies that oxygenated molecular fragments $\left(\mathrm{C}_{x} \mathrm{O}_{y}\right)$ are preferentially incorporated into the film. The boiling temperatures of $\mathrm{C}_{x} \mathrm{O}_{y}$ molecules are typically higher than $\mathrm{C}_{m} \mathrm{~F}_{n}$ molecules. Moreover, it is well known that the deposition rate of a-C:H decreases with increasing substrate temperature. Hence, the increase in the $[\mathrm{C}]$ is not necessarily due to $\mathrm{CD}_{4}$ species. Despite the 
relatively large decrease in the $[\mathrm{F}]$, and the smaller decrease in the [Er], the relative PL intensity slowly decreases as indicated in Figure $3 \mathrm{~b}$. This is attributed to the increasing $[\mathrm{O}] /[\mathrm{Er}]$ ratio as seen in Figure $3 \mathrm{c}$. Hence, more Er atoms are considered to be in the 3+ state. As well, the loss of a large fraction of the fluorinated ligands which in turn have likely been replaced by the deuterated film is considered to suppress the optical quenching of excited $\mathrm{Er}^{3+}$ ions. As the substrate temperature is increased beyond $100{ }^{\circ} \mathrm{C}$, there is an increase in the desorption rate or a lowering of the sticking coefficient of $\mathrm{O}$ containing species. At a substrate temperature of $150{ }^{\circ} \mathrm{C}$, the majority of the film is deuterated amorphous carbon with minute $\mathrm{O}, \mathrm{F}$, and Er concentration. The $[\mathrm{O}] /[\mathrm{Er}]$ ratio is 22.78 and a PL signal is present, although the peak is approximately $7 \times$ lower than the maximum PL signal due to the lower Er concentration. Examining the change in the optical properties of a-C:D host, it is observed that $k$ increases from $1.65 \times 10^{-3}$ to $3.2 \times 10^{-3}$ and $E_{04}$ decreases from 3.81 to $3.41 \mathrm{eV}$ as the substrate temperature is increased from 80 to $150{ }^{\circ} \mathrm{C}$. This minute change is not expected to significantly influence the optical pumping, absorption and PL.

\subsection{Er Quenching Concentration in a-C:D(Er) Film}

In order to study the concentration dependence of the incorporated $\operatorname{Er}(\operatorname{fod})_{3}$ on luminescence of the a-C:D matrix in more detail, the PL intensity which peaks at $1540 \mathrm{~nm}$ is normalized to the film thickness, denoted $I_{\text {nor }}$. Figure $3 \mathrm{~d}$ plots the $I_{\text {nor }}$ as a function of the Er concentration, $N_{\mathrm{Er}}$. $I_{\text {nor }}$ is proportional to $\sigma \varphi N \Gamma / \Gamma_{\mathrm{dar}}$ under the continuous laser pumping condition [25], where $\sigma$ is the excitation cross section, $\varphi$ is the photon flux, $N$ is the optically active Er concentration, $\Gamma$ is the lifetime, and $\Gamma_{\text {dar }}$ is the radiative lifetime. The increase in $N_{\mathrm{Er}}$ will result in a linear increase in $I_{\text {nor }}$ if all the incorporated Er atoms are optically active, i.e., $N \sim N_{\mathrm{Er}}$, and if there are no quenching effects to adversely affect $\sigma$ and $\Gamma / \Gamma_{\text {dar }}$. In Figure $3 \mathrm{~d}$, it is observed that $I_{\text {nor }}$ increases linearly up to an Er concentration of approximately 2.2 at $\%$ and thereafter begins to drop. The latter suggests a reduction in lifetime $\Gamma$ as $N_{\text {Er }}$ increases beyond this point. It indicates that the Er concentration quenching effect sets in after the Er concentration reaches $\sim 2.8$ at $\%$ and continues to increase until 5.5 at $\%$ at which point the PL becomes very weak.

\subsection{Er Oxidation State in a-C:D(Er) Film}

The Er4d XPS spectra of the Er(fod $)_{3}$ powder, the evaporated $\operatorname{Er}(\text { fod })_{3}$ film, and the three a-C:D(Er) samples with different Er concentrations are compared in Figure 4a. The spectra reveal similar spectral characteristic feature at a binding energy of approximately $169.5 \mathrm{eV}$ for all of the samples. This is attributed to the $4 \mathrm{~d}$ levels in the $\mathrm{Er}^{3+}$ ions forming a multiplet through the interaction with the unfilled shell. Hence, the incorporated $\mathrm{Er}$ in a-C:D(Er) films is similar to the $\mathrm{Er}$ in the $\mathrm{Er}(\mathrm{fod})_{3}$ compound. Moreover, it suggests partial preservation of the $\mathrm{Er}^{3+}$ state in a-C:D(Er) films. The presence of a PL signal also supports this result, in spite of the fact that the O/Er ratio is less than 6 as is the case in the pristine $\operatorname{Er}(\mathrm{fod})_{3}$ powder. The depth distribution as depicted in Figure $4 \mathrm{~b}$ reveals a uniform concentration of Er throughout the film of $850 \mathrm{~nm}$ in thickness. This is in contrast to ion-implantation of Er where the optically active ions are always located near the surface [9]. It is noteworthy that the high oxygen concentration at the surface is simply surface contamination. Optimization of process 
parameters and the effect of $\mathrm{F}$ composition on optical properties of the carbon matrix, as well as understanding the electronic transfer mechanism are currently under investigation.

Figure 4. (a) XPS spectra of the three a-C:D(Er) films, $\operatorname{Er}(\mathrm{fod})_{3}$ film (evaporated in the vacuum chamber with $\mathrm{CD}_{4}$ precursor gas flowing without plasma ignition), and the as-received stoichiometric $\operatorname{Er}(\text { fod })_{3}$ powder. The curves have been shifted vertically for clarity of presentation; (b) Depth profile of C, F, O, and Er concentrations in a-C:D(Er) film deposited at $60 \mathrm{~W}$ of RF power and $80{ }^{\circ} \mathrm{C}$ as determined from XPS measurements.

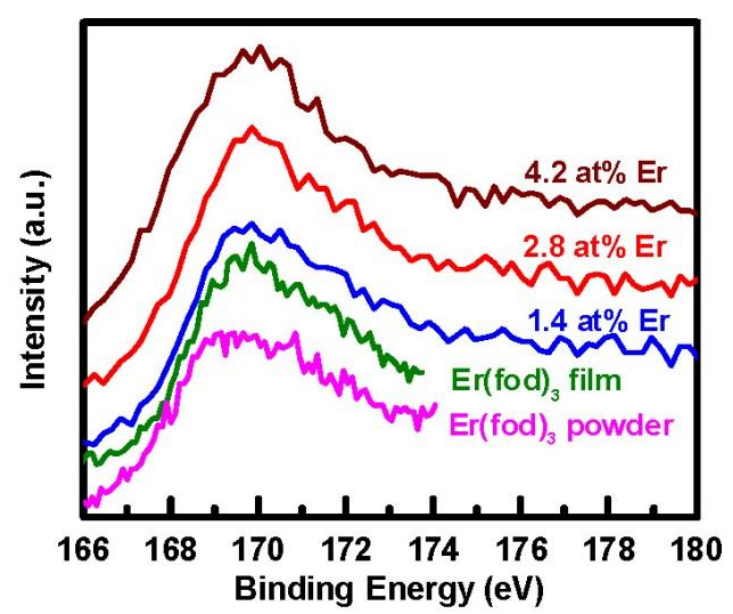

(a)

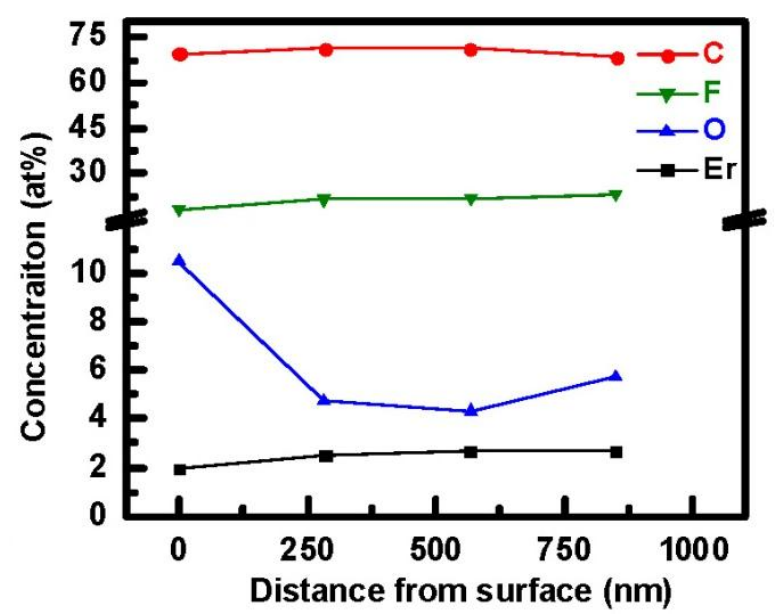

(b)

Currently deuterated methane $\left(\mathrm{CD}_{4}\right)$ gas is more expensive than standard methane $\left(\mathrm{CH}_{4}\right)$ gas. Thus, a path towards device manufacturing with deuteration may include schemes such as the mixing of $\mathrm{CH}_{4}$ and $\mathrm{CD}_{4}$ or $\mathrm{CH}_{4}$ and $\mathrm{D}_{2}$ as the precursor gas. Clearly, optimization of the device processing would be required in order to maintain the PL enhancement. Furthermore, even though the deployment of $\mathrm{CD}_{4}$ leads to PL enhancement in the current deposition method, the ligand of the Er metal-organic compound is in a hydrogenated form. This contrasts the extensive research of PL enhancement by deuterated ligands and/or deuterated polymer host [26,27], where the synthesis procedures are more complicated and could result in higher manufacturing costs.

\section{Experimental Section}

\subsection{RF-PEMOCVD and Sample Preparation}

A capacitively coupled RF-PEMOCVD system, shown in Figure 5, with a base pressure of $5 \times 10^{-5}$ Torr was deployed to deposit Er metal-organic doped a-C thin films. An ac-powered thermal evaporator situated next to the RF-powered electrode (cathode), inside the deposition chamber was utilized to in situ dope the Er metal-organic compound concurrent with the a-C film deposition. A thermocouple is embedded on the bottom of the evaporator, providing feedback to the temperature controller for precise control of the evaporation temperature. A low pass filter is connected using $\mathrm{Cu}$ conductors between the thermal evaporator inside the chamber and the external ac power supply in order to prevent the RF plasma power source from dissipating into the temperature controller or the ac power line. The temperature of the vapor delivery nozzle was kept higher than that of the bottom of the container, by 
$30-50{ }^{\circ} \mathrm{C}$, so as to avoid condensation of the Er metal-organic vapor on the delivery nozzle. The in situ thermal evaporation technique provides the potential of doping Er in a vertically uniform profile as well as design specific concentration profiles. This is unlike the ion-implantation technique, post solution immersion method, or vapor exposure to an Er source procedure [14] where the optically active ions are invariably located near the surface. Further, film damage caused by the high energy ions in the ion-implantation process is avoided. Moreover, the present approach does not require an additional sacrificial metal layer which is typical in the implantation process for non-metallic thin film hosts so as to prevent electrical charging. Also, compared to the co-sputtering [10] or pulsed laser ablation [13,28] of an Er-containing target, this method enables independent control of the Er concentration profile and optical properties of the host material.

Figure 5. Schematic diagram of the RF-PEMOCVD system used for the preparation of a-C:D(Er) films.

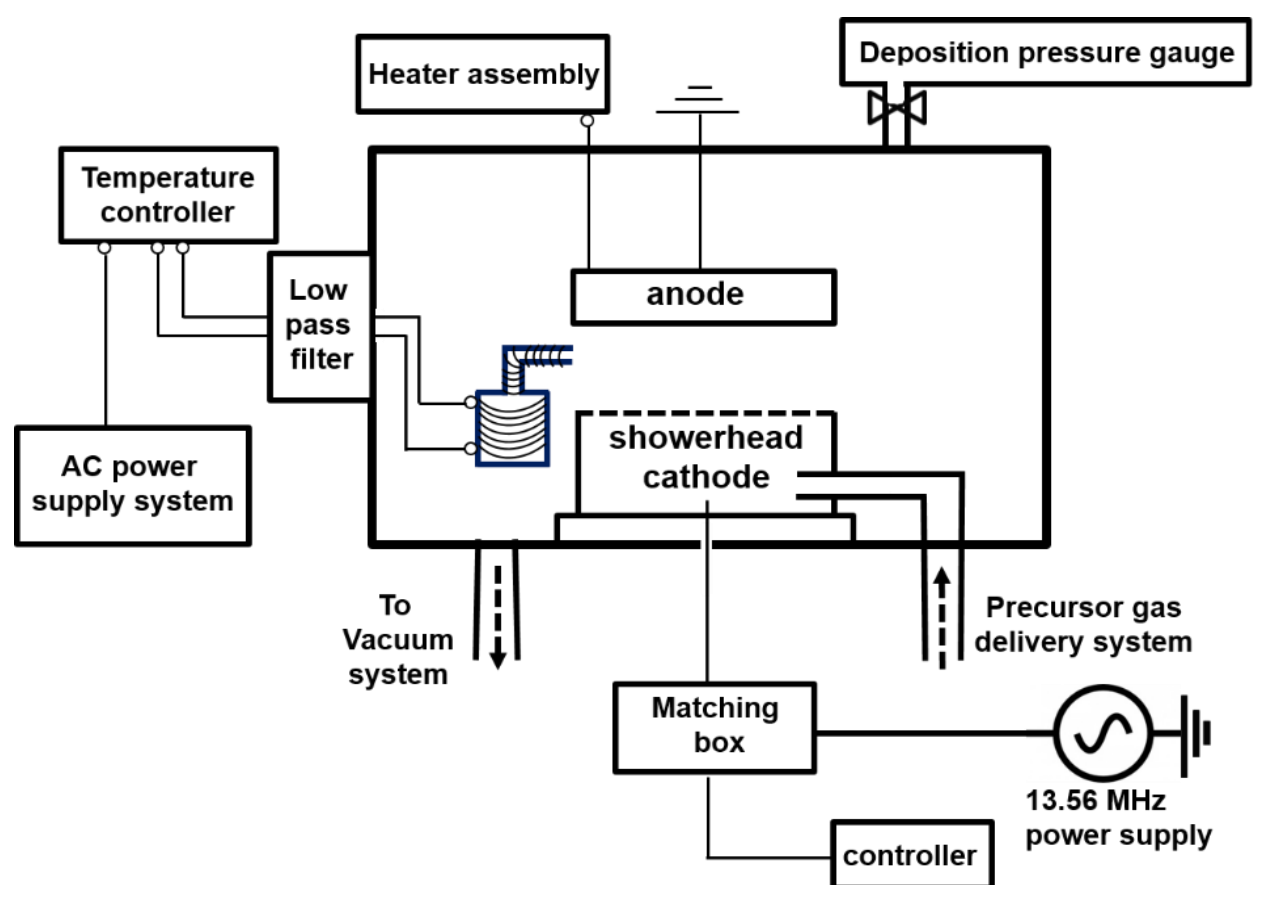

For a-C(Er) samples, the hydrocarbon gas flow rate was $20 \mathrm{sccm}$ and the chamber pressure was $60 \mathrm{mTorr}$ unless otherwise stated. For the films where the RF power was varied, the power was varied from 40 to $60 \mathrm{~W}$ while the substrate temperature was kept constant at $80{ }^{\circ} \mathrm{C}$. For the films where the substrate temperature was varied, the temperature was varied from 80 to $150{ }^{\circ} \mathrm{C}$ while the RF power was kept constant at $60 \mathrm{~W}$. Double-side polished crystalline silicon substrates with resistivity of $30 \mathrm{ohm}-\mathrm{cm}$ were subjected to the standard CMOS cleaning procedure before being loaded into the chamber.

\subsection{Incorporated Er Metal-organic Compound}

The Er metal-organic compound tris (6,6,7,7,8,8,8-heptafluoro-2,2-dimethyl-3,5-octanedionate) Erbium(+III), abbreviated $\left(\mathrm{Er}(\mathrm{fod})_{3}\right)$, having the chemical structure $\mathrm{Er}\left(\mathrm{C}_{10} \mathrm{H}_{10} \mathrm{~F}_{7} \mathrm{O}_{2}\right)_{3}$ and illustrated in Figure $6 \mathrm{a}$, is selected as the doping candidate for a-C(Er) films. It is presumed that the optically active 
$\mathrm{Er}^{3+}$ ions originating from the $\mathrm{Er}(\mathrm{fod})_{3}$ compound can be preserved under appropriate deposition conditions, similar to previous demonstration of other $\mathrm{Er}$ metal-organic compound such as $\mathrm{Er}(\mathrm{thmd})_{3}[29,30]$. Accordingly, these $\mathrm{Er}^{3+}$ ions would not require a high temperature post-deposition annealing step to be optically activated and/or to repair film damage. In a similar compound, tris(2,2,6,6-tetramethyl-3-5 heptanedionato) erbium(+III) powder, denoted $\operatorname{Er}(\operatorname{tmhd})_{3}, \quad \operatorname{Er}$ is coordinated to six oxygen atoms. It has been demonstrated that the Er local environment in an as-deposited a-Si:H(Er) sample prepared by PECVD is very similar to $\mathrm{Er}_{2} \mathrm{O}_{3}$. This bonding environment has efficiently promoted the optically emitting centers [30]. In this work, a controlled vapor flux of $\mathrm{Er}$ (fod) $)_{3}$ compound is introduced by thermal evaporation at $150{ }^{\circ} \mathrm{C}$. The vapor flux is mixed with the hydrocarbon plasma for a-C(Er) film formation. In order to determine the proper wavelength of the pumping source for the $\operatorname{Er}(\text { fod })_{3}$ compound, the absorption spectrum was collected with an UV-Vis-NIR spectrometer (PerkinElmer, Waltham, MA, USA). As displayed in Figure 6b, the absorption spectrum for the $\operatorname{Er}(\text { fod })_{3}$ compound dissolved in a d-chloroform solvent suggests that the potential excitation source can be near $520 \mathrm{~nm}$.

Another advantage of the $\operatorname{Er}(\text { fod })_{3}$ compound deployed in this work is the partial fluorination of the hydrogen-containing ligands which is expected to reduce the $\mathrm{C}-\mathrm{H}$ quenching effect. The ligands and coordinated solvent molecules of Er metal-organic compounds usually contain $\mathrm{C}-\mathrm{H}$ and $\mathrm{O}-\mathrm{H}$ bonds. Accordingly, if excited $\mathrm{Er}^{3+}$ ions are disturbed nearby $\mathrm{C}-\mathrm{H}$ and $\mathrm{O}-\mathrm{H}$ oscillators, a non-radiative transition occurs [23]. This would dramatically reduce the luminescence efficiency. It has also been quantitatively demonstrated that a three order of magnitude increase in the radiative lifetime can be achieved by increasing the distance between the neighboring $\mathrm{C}-\mathrm{H}$ and $\mathrm{O}-\mathrm{H}$ bonds in the ligands and the $\mathrm{Er}^{3+}$ ion [31]. However, the presence of $\mathrm{C}-\mathrm{H}$ vibrational oscillators within a sphere of at least $20 \AA$ from the $\mathrm{Er}^{3+}$ ion can still be an effective de-excitation site [31]. Thus, fluorination of the hydrogen-containing ligands is expected to reduce the non-radiative deactivation channels due to $\mathrm{C}-\mathrm{H}$ bonds. This will enable an enhancement in the $\mathrm{Er}^{3+}$ luminescence efficiency [27,32].

Figure 6. (a) Illustration of the Er metal-organic compound, $\operatorname{Er}(\text { fod })_{3}$, with chemical structure $\operatorname{Er}\left(\mathrm{C}_{10} \mathrm{H}_{10} \mathrm{O}_{2} \mathrm{~F}_{7}\right)_{3}$. The large center blue atom represents $\mathrm{Er}$, red atoms represent $\mathrm{O}$, dark grey atoms represent $\mathrm{C}$, yellow atoms represent $\mathrm{F}$, and white atoms represent $\mathrm{H}$; (b) The absorption spectra of Er(fod $)_{3}$ metal-organic compound dissolved in d-chloroform solvent.

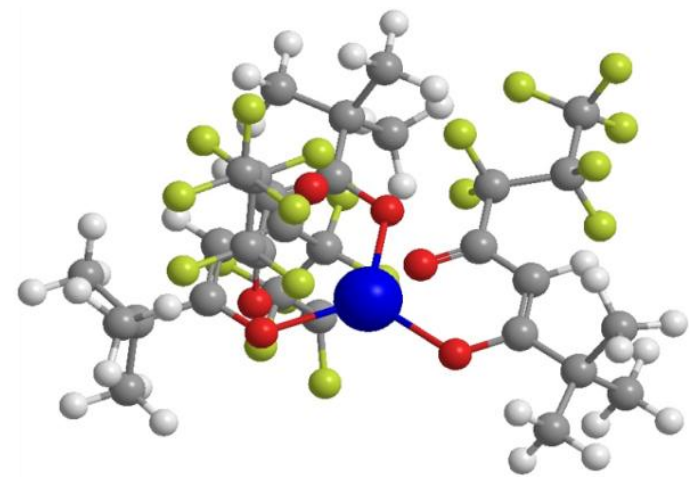

(a)

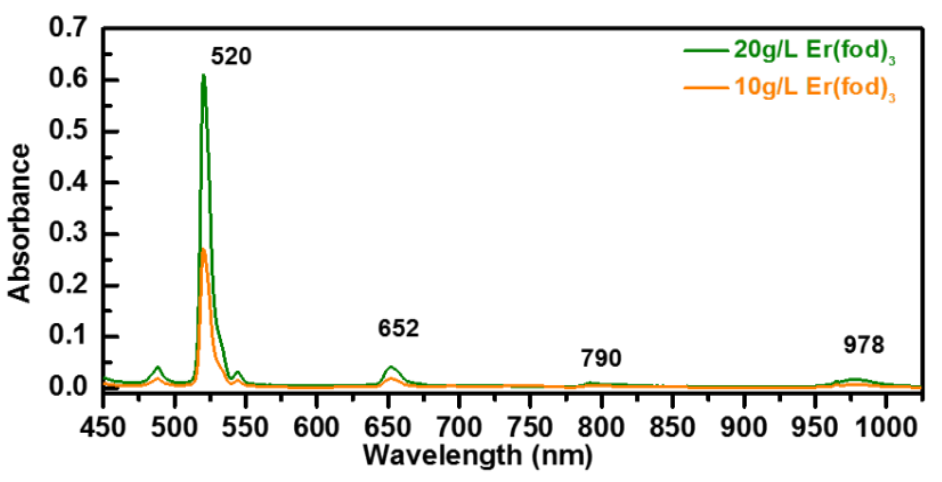

(b) 


\subsection{Film Characterization}

\subsubsection{Photoluminescence}

Photoluminescence spectra of Er metal-organic doped a-C based films were collected at room temperature to verify the optical activity of $\mathrm{Er}$ in the a-C films. A continuous wave diode-pumped solid-state $532 \mathrm{~nm}$ laser with a power density of $80 \mathrm{~mW} / \mathrm{mm}^{2}$ was used as the excitation source, in keeping with the observed absorption peak for the $\operatorname{Er}(\text { fod })_{3}$ shown in Figure $6 \mathrm{~b}$. A thermoelectrically cooled InGaAs photodiode, 800-1700 nm detection range, with standard lock-in techniques was employed. A single pass monochromator was utilized to disperse the emitted PL. The energy of the laser is near resonance with the ${ }^{4} S_{3 / 2}$ excited level of Er ions. The excited Er ions decay to ${ }^{4} I_{13 / 2}$ level through the fast non-radiative transition and then emit at $1540 \mathrm{~nm}$ through ${ }^{4} I_{13 / 2}$ to ${ }^{4} I_{15 / 2}$ transition.

\subsubsection{X-Ray Photoelectron Spectroscopy}

X-ray photoelectron spectroscopy (XPS) was employed to quantitatively characterize the elemental composition and the depth distribution of a-C(Er) films. The XPS spectra were collected from the surface of the sample using a monochromatic Al Ka X-ray source in a Thermo Scientific K-Alpha spectrometer (ThermoFisher Scientific, Waltham, MA, USA) with an ultrahigh vacuum of the order of $10^{-9}$ Torr. The samples were attached to a stainless steel holder using $\mathrm{Cu}$ conductive double-sided tape. The top of the sample was also grounded by $\mathrm{Cu}$ tape in order to prevent severe charging effects.

\subsubsection{Spectroscopic Ellipsometry and Fourier Transform InfraRed Spectroscopy}

The extinction coefficient $k$ and optical bandgap $E_{04}$ of the host deuterated amorphous carbon (a-C:D) films were probed through spectroscopic ellipsometry. The measurements were carried out using an UV-Vis-NIR spectroscopic ellipsometer (Sopralab, Paris, France). The wavelength range was $350-1700 \mathrm{~nm}$ at an incident angle of $75^{\circ}$. The spectra were analyzed by regression fitting using the linear Levenberg-Marquard algorithm method with a maximum of 1000 iterations using a three-layer optical structure comprising void (ambient)/a-C layer/c-Si substrate. A first-order initial thickness approximation of the a-C film was estimated from profilometry measurements. The five constants of Forouhi-Bloomer dispersion model [33] and thickness of the a-C:D layer were allowed to vary during the fitting process. The optical bandgap $E_{04}$, defined as the photon energy at which the absorption coefficient $\alpha(=4 \pi k / \lambda)$ is equal to $10^{4} \mathrm{~cm}^{-1}$, was determined from the extinction coefficient $k$; $\lambda$ is the wavelength. The fits yielded a coefficient of regression $\mathrm{R}^{2} \sim 0.99$ and the error of the six fitting parameters was less than $+/-10 \%$, indicating the model was appropriate for the a-C:D films.

The absorption spectra of the host a-C films were characterized by Fourier Transform InfraRed (FTIR) Spectroscopy using a Perkin Elmer 2000 spectrometer (PerkinElmer, Waltham, MA, USA) with a resolution of $4 \mathrm{~cm}^{-1}$. The transmission spectra were background corrected for the interference patterns emerging due to multiple reflections in the film. The absorption was determined using the following relation:

$$
\alpha(v)=-\frac{1}{d} \ln T
$$


where $\alpha(v)$ is the absorption constant, $d$ is the thickness of the film, and $T$ is the normalized transmission of the a-C film with the background removed.

\section{Conclusions}

The feasibility of the in situ growth of Er-doped a-C thin films on Si substrates at low temperature $\left(<200{ }^{\circ} \mathrm{C}\right)$ by a simple one-step metal-organic radio frequency plasma-enhanced chemical vapor deposition system was successfully demonstrated. By adopting a new Er metal-organic precursor with partial fluorination, $\mathrm{Er}(\mathrm{fod})_{3}$, the optically active $\mathrm{Er}^{3+}$ ions are conserved, thus avoiding a subsequent high temperature annealing procedure and accordingly resulting in room temperature luminescence. Furthermore, the enhancement of the PL was demonstrated via deuteration of the a-C host by effectively shifting the quenching vibrational modes to lower wavenumbers and decreasing the respective magnitudes of absorption. The effect of RF power and substrate temperature on the Er concentration, $[\mathrm{O}] /[\mathrm{Er}]$ ratio, and the respective PL intensity of the a-C:D(Er) film was investigated. It was observed that the PL signal increases with increasing RF power, which is attributed to an increase in $[\mathrm{O}] /[\mathrm{Er}]$ ratio and hence the erbium-oxygen coordination number. The relatively large $[\mathrm{F}]$ is deemed to contribute to the enhancement in PL efficiency. In addition, PL intensity and [Er] decrease with increasing substrate temperature; the decrease in $[\mathrm{Er}]$ is attributed to an increased desorption rate or a lower sticking coefficient of the fluorinated fragments during film growth. By examining the relationship of the normalized PL intensity, $I_{\text {nor }}$, as a function of the Er concentration, it is observed that Er concentration quenching begins at $\sim 2.2$ at $\%$ and continues to increase until 5.5 at $\%$ at which point the PL is very weak. Furthermore, the foregoing indicate the preservation of the $\mathrm{Er}^{3+}$ state in a-C:D(Er) films was achieved. The in situ thermal evaporation technique provides the capability of doping Er in a uniform profile vertically/depth-wise.

\section{Acknowledgments}

This work was supported by the Natural Sciences and Engineering Research Council of Canada (NSERC) Discovery grants, the NSERC CREATE program in Nanoscience and Nanotechnology, and the Departments of Electrical and Computer Engineering and Materials Science and Engineering at the University of Toronto. The authors also acknowledge valuable discussions with Rana Sodhi for the analysis of XPS results, and help from Davit Yeghikyan and Tome Kosteski for the assembly of RF-PEMOCVD system.

\section{Author Contributions}

Hui-Lin Hsu and Keith R. Leong planned and conducted most of the experiments related to this study under the supervision of Prof. Li Qian and Prof. Nazir P. Kherani. The principal contributions of I-Ju Teng, Jenh-Yih Juang and Sheng-Rui Jian comprised photoluminescence and XPS characterizations and analyses. Michael Halamicek assisted with the Spectroscopic Ellipsometry and Fourier Transform InfraRed Spectroscopy measurements and analyses. The article was written and edited by Hui-Lin Hsu, Keith R. Leong, Li Qian, and Nazir P. Kherani. 


\section{Conflicts of Interest}

The authors declare no conflict of interest.

\section{References}

1. Paniccia, M. Integrating silicon photonics. Nat. Photon. 2010, 4, 498-499.

2. Xiao, H. Introduction to Semiconductor Manufacturing Technology; SPIE Press: Bellingham, WA, USA, 2012.

3. Pavesi, L.; Dal Negro, L.; Mazzoleni, C.; Franzo, G.; Priolo, F. Optical gain in silicon nanocrystals. Nature 2000, 408, 440-444.

4. Rong, H.; Liu, A.; Jones, R.; Cohen, O.; Hak, D.; Nicolaescu, R.; Fang, A.; Paniccia, M. An all-silicon Raman laser. Nature 2005, 433, 292-294.

5. Green, M.A.; Zhao, J.; Wang, A.; Reece, P.J.; Gal, M. Efficient silicon light-emitting diodes. Nature 2001, 412, 805-808.

6. Walters, R.J.; Bourianoff, G.I.; Atwater, H.A. Field-effect electroluminescence in silicon nanocrystals. Nat. Mater. 2005, 4, 143-146.

7. Xu, Q.; Schmidt, B.; Pradhan, S.; Lipson, M. Micrometre-scale silicon electro-optic modulator. Nature 2005, 435, 325-327.

8. Miritello, M.; Lo Savio, R.; Iacona, F.; Franzò, G.; Irrera, A.; Piro, A.M.; Bongiorno, C.; Priolo, F. Efficient luminescence and energy transfer in erbium silicate thin films. Adv. Mater. 2007, 19, 1582-1588.

9. Polman, A. Erbium implanted thin film photonic materials. J. Appl. Phys. 1997, 82, 1-39.

10. Weiser, G.; Kühne, H.; Terukov, I.E. Energy transfer to $\mathrm{Er}^{3+}$ ions in a-Si ${ }_{1-x} \mathrm{C}_{x}: \mathrm{H}$ alloys: Emission at 1.54 mm wavelength. Phys. Status Solidi C 2004, 1, 1275-1283.

11. Priolo, F.; Franzo, G.; Pacifici, D.; Vinciguerra, V.; Iacona, F.; Irrera, A. Role of the energy transfer in the optical properties of undoped and Er-doped interacting Si nanocrystals. J. Appl. Phys. 2006, 89, 264-272.

12. Baranov, A.M.; Sleptsov, V.V.; Nefedov, A.A.; Varfolomeev, A.E.; Fanchenko, S.S.; Calliari, L.; Speranza, G.; Ferrari, M.; Chiasera, A. Erbium photoluminescence in hydrogenated amorphous carbon. Phys. Status Solidi B 2002, 234, R1-R3.

13. Foong, Y.M.; Hsieh, J.; Li, X.; Chua, D.H.C. The study on the effect of erbium on diamond-like carbon deposited by pulsed laser deposition technique. J. Appl. Phys. 2009, 106, 064904:1-064904:8.

14. Prajzler, V.; Huttel, I.; Nekvindova, P.; Schrofel, J.; Mackova, A.; Gurovic, J. Erbium doping into thin carbon optical layers. Thin Solid Films 2003, 433, 363-366.

15. Speranza, G.; Calliari, L.; Ferrari, M.; Chiasera, A.; Tran Ngoc, K.; Baranov, A.M.; Sleptsov, V.V.; Nefedov, A.A.; Varfolomeev, A.E.; Fanchenko, S.S. Erbium-doped thin amorphous carbon films prepared by mixed CVD sputtering. Appl. Surf. Sci. 2004, 238, 117-120.

16. Tsai, R.Y.C.; Qian, L.; Alizadeh, H.; Kherani, N.P. Room-temperature photoluminescence in erbium-doped deuterated amorphous carbon prepared by low-temperature MO-PECVD. Opt. Express 2009, 17, 21098-21107. 
17. McLaughlin, J.A.; Maguire, P.D. Advances on the use of carbon based materials at the biological and surface interface for applications in medical implants. Diam. Relat. Mater. 2008, 17, 873-877.

18. Patsalas, P. Optical properties of amorphous carbons and their applications and perspectives in photonics. Thin Solid Films 2011, 519, 3990-3996.

19. Robertson, J. Diamond-like amorphous carbon. Mater. Sci. Eng. R Rep. 2002, 37, 129-281.

20. Piazza, F.; Grambole, D.; Schneider, D.; Casiraghi, C.; Ferrari, A.C.; Robertson, J. Protective diamond-like carbon coatings for future optical storage disks. Diam. Relat. Mater. 2005, 14, 994-999.

21. Wong, W.H.; Chan, K.S.; Pun, E.Y.B. Ultraviolet direct printing of rare-earth-doped polymer waveguide amplifiers. Appl. Phys. Lett. 2005, 87, 011103:1-011103:3.

22. Wong, W.H.; Pun, E.Y.B.; Chan, K.S. $\mathrm{Er}^{3+}-\mathrm{Yb}^{3+}$ codoped polymeric optical waveguide amplifiers. Appl. Phys. Lett. 2004, 84, 176-178.

23. Haas, Y.; Stein, G.; Wurzberg, E. Radiationless transitions in solutions: Isotope and proximity effects on $\mathrm{Dy}^{3+}$ by $\mathrm{C}-\mathrm{H}$ and $\mathrm{C}-\mathrm{N}$ bonds. J. Chem. Phys. 1974, 60, 258-263.

24. Siebrand, W. Radiationless transitions in polyatomic molecules. I. Calculation of Franck-Condon Factors. J. Chem. Phys. 1967, 46, 440-447.

25. Saleh, B.E.A.; Teich, M.C. Fundamentals of Photonics; Wiley: Hoboken, NJ, USA, 2007.

26. Hasegawa, Y.; Wada, Y.; Yanagida, S. Strategies for the design of luminescent lanthanide(III) complexes and their photonic applications. J. Photochem. Photobiol. C 2004, 5, 183-202.

27. Kuriki, K.; Koike, Y.; Okamoto, Y. Plastic optical fiber lasers and amplifiers containing lanthanide complexes. Chem. Rev. 2002, 102, 2347-2356.

28. Foong, Y.M.; Hsieh, J.; Li, X.; Chua, D.H.C. Comparative study between erbium and erbium oxide-doped diamondlike carbon films deposited by pulsed laser deposition technique. J. Vac. Sci. Technol. A 2010, 28, 449-455.

29. Terukov, E.I.; Konkov, O.I.; Kudoyarova, V.K.; Koughia, K.V.; Weiser, G.; Kühne, H.; Kleider, J.P.; Longeaud, C.; Brüggemann, R. Erbium incorporation in plasma-deposited amorphous silicon. J. Noncryst. Solids 2000, 266-269, 614-618.

30. Cínthia Piamonteze, L.R.T.; Hélio Tolentino, M.C.M.A.; Gerhard, W.; Eugeny, T. Er environment in a-Si:H(Er) prepared by PECVD. Mater. Res. Soc. Symp. Proc. 2000, 609, doi:10.1557/PROC-609-A11.2.

31. Winkless, L.; Tan, R.H.C.; Zheng, Y.; Motevalli, M.; Wyatt, P.B.; Gillin, W.P. Quenching of Er(III) luminescence by ligand $\mathrm{C}-\mathrm{H}$ vibrations: Implications for the use of erbium complexes in telecommunications. Appl. Phys. Lett. 2006, 89, 111115:1-111115:3.

32. Monguzzi, A.; Tubino, R.; Meinardi, F.; Biroli, A.O.; Pizzotti, M.; Demartin, F.; Quochi, F.; Cordella, F.; Loi, M.A. Novel $\mathrm{Er}^{3+}$ perfluorinated complexes for broadband sensitized near infrared emission. Chem. Mater. 2008, 21, 128-135.

33. Forouhi, A.R.; Bloomer, I. Optical dispersion-relations for amorphous-semiconductors and amorphous dielectrics. Phys. Rev. B 1986, 34, 7018-7026.

(C) 2014 by the authors; licensee MDPI, Basel, Switzerland. This article is an open access article distributed under the terms and conditions of the Creative Commons Attribution license (http://creativecommons.org/licenses/by/3.0/). 